\title{
Quadruple Bordered Constructions of Self-Dual Codes from Group Rings
}

\author{
Steven T. Dougherty * \\ Department of Mathematics University of Scranton \\ Scranton, PA 18510 \\ USA \\ Joseph Gildea ${ }^{\dagger}$, \\ University of Chester \\ Department of Mathematics \\ Chester, UK \\ Abidin Kaya ${ }^{\ddagger}$ \\ Sampoerna Academy, L'Avenue Campus \\ 12780, Jakarta, Indonesia
}

April 2, 2019

\begin{abstract}
In this paper, we introduce a new bordered construction for self-dual codes using group rings. We consider constructions over the binary field, the family of rings $R_{k}$ and the ring $\mathbb{F}_{4}+u \mathbb{F}_{4}$. We use groups of order 4, 12 and 20. We construct some extremal self-dual codes and non-extremal self-dual codes of length 16, 32, 48, 64 and 68. In particular, we construct 33 new extremal self-dual codes of length 68 .
\end{abstract}

Key Words: Group rings; self-dual codes; codes over rings; extremal codes; bordered constructions.

*steven.dougherty@scranton.edu,prof.steven.dougherty@gmail.com

†J.Gildea@chester.ac.uk

†abidinkaya@gmail.com 


\section{Introduction}

Self-dual codes are one of the most widely studied families of codes. There are many reasons for this including their connection to designs and lattices as well as their importance as codes. Numerous techniques have been given to find self-dual codes and a great deal of attention has been paid to determining the existence of extremal self-dual codes, that is codes meeting a bound which comes from the application of invariant theory. In this paper, we shall show why new techniques are needed and we shall give a new construction which produces many interesting codes.

The techniques that we shall describe for constructing self-dual codes are generally concerned with circulant matrices. We recall the definition of a circulant matrix.

Definition 1. A circulant matrix over a ring $R$ is a square $n \times n$ matrix, which takes the form

$$
\operatorname{circ}\left(a_{1}, a_{2}, \ldots, a_{n}\right)=\left(\begin{array}{ccccc}
a_{1} & a_{2} & a_{3} & \ldots & a_{n} \\
a_{n} & a_{1} & a_{2} & \ldots & a_{n-1} \\
a_{n-1} & a_{n} & a_{1} & \ldots & a_{n-2} \\
\vdots & \vdots & \vdots & \ddots & \vdots \\
a_{2} & a_{3} & a_{4} & \ldots & a_{1}
\end{array}\right)
$$

where $a_{i} \in R$. A reverse circulant matrix over a ring $R$ is a square $n \times n$ matrix, which takes the form

$$
\operatorname{rcirc}\left(a_{1}, a_{2}, \ldots, a_{n}\right)=\left(\begin{array}{ccccc}
a_{1} & a_{2} & a_{3} & \ldots & a_{n} \\
a_{2} & a_{3} & a_{4} & \ldots & a_{1} \\
a_{3} & a_{4} & a_{5} & \ldots & a_{2} \\
\vdots & \vdots & \vdots & \ddots & \vdots \\
a_{n} & a_{1} & a_{2} & \ldots & a_{n-1}
\end{array}\right)
$$

where $a_{i} \in R$.

The most widely applied construction for self-dual codes is to use matrices of the form $\left(I_{n} \mid A\right)$ where $A$ is a circulant matrix satisfying $A A^{T}=-I_{n}$. Numerous combinatorial and algebraic techniques have been applied to this case to produce interesting circulant matrices.

This technique, known as the double circulant construction was first introduced in the 1960s, (see [3, 25] for example). This method has been used extensively to create self-dual codes since it's inception (see [16, 17, 18, 19, 20] for example). MacWilliams and Sloane ([26]) extended this technique to the single bordered construction where the matrix $A$ is replaced with

$$
\left(\begin{array}{c|ccc}
\gamma & \alpha & \ldots & \alpha \\
\hline \alpha & & & \\
\vdots & & B & \\
\alpha & &
\end{array}\right)
$$


where $B$ is a circulant matrix.

In [24], Kaya et. al. modified the pure circulant construction where $A$ is replaced with

$$
\left(\begin{array}{cc|cc}
1 & 1 & \mathbf{x} & \mathbf{y} \\
1 & 1 & \mathbf{y} & \mathbf{x} \\
\hline \mathbf{z}^{\mathbf{T}} & \mathbf{t}^{\mathbf{T}} & A & B \\
\mathbf{t}^{\mathbf{T}} & \mathbf{z}^{\mathbf{T}} & B & A
\end{array}\right)
$$

where $A$ is an $n \times n$ circulant matrix, $B$ is a $n \times n$ reverse circulant matrix and $\mathbf{y}, \mathbf{x}, \mathbf{z}, \mathbf{t}$ are vectors of length $n$. In this work, we shall extend the double circulant construction where $A$ is replaced with the matrix:

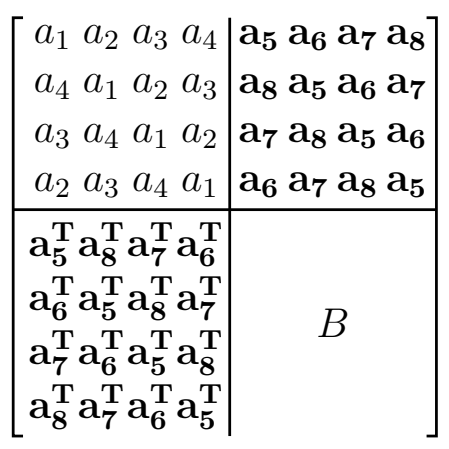

where $\mathbf{a}_{\mathbf{5}}, \mathbf{a}_{\mathbf{6}}, \mathbf{a}_{\mathbf{7}}$ and $\mathbf{a}_{\mathbf{8}}$ are vectors of length $n$. In particular, we will consider matrices $B$ that arise from a group ring construction.

Group rings have been used in the literature to construct self-dual codes using a different technique. In [1], an ideal of the group algebra $\mathbb{F}_{2} S_{4}$ was used to construct the famous binary extended Golay code where $\mathbb{F}_{2}$ is the Galois field of 2 elements and $S_{4}$ is the symmetric group on 4 elements. In [22], an isomorphism between a group ring and a certain subring of the $n \times n$ matrices over the ring was established. This isomorphism was used to produce self-dual codes in [23, 30]. In [29], McLoughlin found that the $[48,24,12]$ Type II code is a dihedral code. In $[6,8]$, the idea was extended to any group $G$ and $G$-codes were defined as codes that are ideals in the group $\operatorname{ring} R G$, where $R$ is a finite Frobenius ring. In [15], a connection between certain group ring elements called unitary units and self-dual codes was established (under a certain construction). Under this construction, it was also highlighted that certain well established techniques are naturally derived from group rings.

In the following sections, we will provide important concepts required for later sections. We will then introduce a new construction and some associated theory. We will finish the article with the implementation of this technique to find certain known and unknown selfdual codes using MAGMA (see [27] for a complete description of this computer algebra system). 


\section{Preliminaries}

In this section, we will define self-dual codes over Frobenius rings of characteristic 2. We will introduce a family of rings called $R_{k}$ and the ring $\mathbb{F}_{4}+u \mathbb{F}_{4}$. This section concludes with an introduction to group rings and an established isomorphism between a group ring and a certain subring of the $n \times n$ matrices over a ring.

\subsection{Self-Dual Codes}

Throughout this paper, all rings are assumed to be commutative, finite, Frobenius rings with a multiplicative identity. A complete description of Frobenius rings can be found in [6].

A code $C$ over a finite commutative ring $R$ is said to be any subset of $R^{n}$. If the code is a submodule of the ambient space then the code is said to be linear. We attach the usual inner-product to the ambient space, namely $[\mathbf{v}, \mathbf{w}]=\sum v_{i} w_{i}$. The orthogonal with respect to this inner-product is defined as $C^{\perp}=\left\{\mathbf{w} \mid \mathbf{w} \in R^{n},[\mathbf{w}, \mathbf{w}]=0, \forall \mathbf{v} \in C\right\}$. The orthogonal code $C^{\perp}$ is linear whether or not $C$ is. Since the ring is Frobenius we have that for all linear codes over $R,|C|\left|C^{\perp}\right|=|R|^{n}$. The proof of this fact and a complete description of codes over rings can be found in $[6]$.

If a code satisfies $C=C^{\perp}$ then the code $C$ is said to be self-dual. If $C \subseteq C^{\perp}$ then the code is said to be self-orthogonal. It follows immediately from the fact that $|C|\left|C^{\perp}\right|=|R|^{n}$, that if $C$ is a self-dual code of length $n$ over the finite commutative Frobenius ring $R$ then $|C|=|R|^{\frac{n}{2}}$. For binary codes, a self-dual code where all weights are congruent to $0(\bmod 4)$ is said to be Type II and the code is said to be Type I otherwise. The bounds on the minimum distances for self-dual codes are:

Theorem 2.1. ([31]) Let $d_{I}(n)$ and $d_{I I}(n)$ be the minimum distance of a Type I and Type II binary code of length $n$, respectively. Then

$$
d_{I I}(n) \leq 4\left\lfloor\frac{n}{24}\right\rfloor+4
$$

and

$$
d_{I}(n) \leq\left\{\begin{array}{lll}
4\left\lfloor\frac{n}{24}\right\rfloor+4 & \text { if } n \not \equiv 22 & (\bmod 24) \\
4\left\lfloor\frac{n}{24}\right\rfloor+6 & \text { if } n \equiv 22 & (\bmod 24)
\end{array}\right.
$$

Self-dual codes that meet these bounds are called extremal. The search for extremal codes is one of the major open questions of algebraic coding theory.

\section{$2.2 \quad$ A Family of Rings}

We now define a family of rings of characteristic 2 which we shall use in our constructions. These rings are denoted by $R_{k}$, for $k \geq 1$, and were defined in [12] and [13]. For $k \geq 1$, 
define

$$
R_{k}=\mathbb{F}_{2}\left[u_{1}, u_{2}, \ldots, u_{k}\right] /\left\langle u_{i}^{2}=0, u_{i} u_{j}=u_{j} u_{i}\right\rangle .
$$

We can also define the rings recursively as:

$$
R_{k}=R_{k-1}\left[u_{k}\right] /\left\langle u_{k}^{2}, u_{k} u_{j}-u_{j} u_{k}\right\rangle=R_{k-1}+u_{k} R_{k-1} .
$$

For any subset $A \subseteq\{1,2, \ldots, k\}$ we will fix

$$
u_{A}:=\prod_{i \in A} u_{i}
$$

with the convention that $u_{\emptyset}=1$. Then any element of $R_{k}$ can be represented as

$$
\sum_{A \subseteq\{1, \ldots, k\}} c_{A} u_{A}, \quad c_{A} \in \mathbb{F}_{2}
$$

An advantage of representing elements with this notation is that we can easily observe that

$$
u_{A} u_{B}= \begin{cases}0 & \text { if } A \cap B \neq \emptyset \\ u_{A \cup B} & \text { if } A \cap B=\emptyset .\end{cases}
$$

This leads to

$$
\left(\sum_{A} c_{A} u_{A}\right)\left(\sum_{B} d_{B} u_{B}\right)=\sum_{A, B \subseteq\{1, \ldots, k\}, A \cap B=\emptyset} c_{A} d_{B} u_{A \cup B} .
$$

It is shown in [12] that the ring $R_{k}$ is a commutative Frobenius ring with $\left|R_{k}\right|=2^{\left(2^{k}\right)}$. The ring is a local ring with maximal ideal $\left\langle u_{1}, u_{2}, \ldots, u_{k}\right\rangle$. The proof of the following lemma can be found in $[12]$.

Lemma 2.2. An element $\gamma$ of $R_{k}$ if and only if $\gamma^{2}=1$. An element $\alpha$ of $R_{k}$ is a non-unit if and only if $\alpha^{2}=0$.

We note then that if $\mathfrak{m}$ is the maximal ideal of $R_{k}$ then $\alpha \in \mathfrak{m}$ if and only if $\alpha$ is a non-unit. Therefore, if $\alpha \in \mathfrak{m}$ then $\alpha^{2}=0$ and if $\alpha \notin \mathfrak{m}$ then $\alpha^{2}=1$.

We shall now given the definition of a Gray map from $R_{k}$ to $\mathbb{F}_{2}^{2^{k}}$, which was previously defined in [12]. For $R_{1}$ we have the following map: $\phi_{1}\left(a+b u_{1}\right)=(b, a+b)$. Then let $c \in R_{k}$, $c$ can be written as $c=a+b u_{k}, a, b \in R_{k-1}$. Then

$$
\phi_{k}(c)=\left(\phi_{k-1}(b), \phi_{k-1}(a+b)\right)
$$

The map $\phi_{k}$ is a distance preserving map and the following is shown in [13].

Theorem 2.3. Let $C$ be a self-dual code over $R_{k}$, then $\phi_{k}(C)$ is a binary self-dual code of length $2^{k} n$. 
The next result which was introduced in [11] proves very useful when extending codes over $R_{1}$.

Theorem 2.4. Let $\mathcal{C}$ be a self-dual code over $R_{k}$ of length $n$ and $G=\left(r_{i}\right)$ be a $j \times n$ generator matrix for $\mathcal{C}$, where $r_{i}$ is the $i$-th row of $G, 1 \leq i \leq k$. Let $c$ be a unit in $R_{k}$ and $X$ be a vector in $R_{k}{ }^{n}$ with $\langle X, X\rangle=1$. Let $y_{i}=\left\langle r_{i}, X\right\rangle$ for $1 \leq i \leq k$. Then the following matrix

$$
\left(\begin{array}{cc|c}
1 & 0 & X \\
\hline y_{1} & c y_{1} & r_{1} \\
\vdots & \vdots & \vdots \\
y_{k} & c y_{k} & r_{k}
\end{array}\right),
$$

generates a self-dual code $\mathcal{C}^{\prime}$ over $R_{k}$ of length $n+2$.

\subsection{The ring $\mathbb{F}_{4}+u \mathbb{F}_{4}$}

We shall now define the ring $\mathbb{F}_{4}+u \mathbb{F}_{4}$, which we shall also use to construct self-dual codes. Let $\mathbb{F}_{4}=\mathbb{F}_{2}(\omega)$ be the quadratic field extension of $\mathbb{F}_{2}$, where $\omega^{2}+\omega+1=0$. The ring $\mathbb{F}_{4}+u \mathbb{F}_{4}$ is defined as $\mathbb{F}_{4}[u] /\left\langle u^{2}\right\rangle$. Note that $\mathbb{F}_{4}+u \mathbb{F}_{4}$ can be viewed as an extension of $\mathbb{F}_{2}+u \mathbb{F}_{2}$ and so we can describe any element of $\mathbb{F}_{4}+u \mathbb{F}_{4}$ in the form $\omega a+\bar{\omega} b$ uniquely, where $a, b \in \mathbb{F}_{2}+u \mathbb{F}_{2}$. In order to fit the upcoming tables we use hexadecimals to denote the elements of $\mathbb{F}_{4}+u \mathbb{F}_{4}$ with the ordered basis $\{u \omega, \omega, u, 1\}$.

A code $C$ of length $n$ over $\mathbb{F}_{4}+u \mathbb{F}_{4}$ is an $\left(\mathbb{F}_{4}+u \mathbb{F}_{4}\right)$-submodule of $\left(\mathbb{F}_{4}+u \mathbb{F}_{4}\right)^{n}$. In [14] and $[7]$ the following Gray maps were introduced:

$$
\begin{aligned}
& \psi_{\mathbb{F}_{4}}: \mathbb{F}_{4}^{n} \rightarrow \mathbb{F}_{2}^{2 n} \quad \mid \varphi_{\mathbb{F}_{2}+u \mathbb{F}_{2}}:\left(\mathbb{F}_{2}+u \mathbb{F}_{2}\right)^{n} \rightarrow \mathbb{F}_{2}^{2 n} \\
& a \omega+b \bar{\omega} \mapsto(a, b), a, b \in \mathbb{F}_{2}^{n} \| a+b u \mapsto(b, a+b), a, b \in \mathbb{F}_{2}^{n} .
\end{aligned}
$$

Those were generalized to the following maps in [28]:

$$
\begin{aligned}
& \psi_{\mathbb{F}_{4}+u \mathbb{F}_{4}}:\left(\mathbb{F}_{4}+u \mathbb{F}_{4}\right)^{n} \rightarrow\left(\mathbb{F}_{2}+u \mathbb{F}_{2}\right)^{2 n} \\
& a \omega+b \bar{\omega} \mapsto(a, b), \quad a, b \in\left(\mathbb{F}_{2}+u \mathbb{F}_{2}\right)^{n}
\end{aligned} \begin{aligned}
& \varphi_{\mathbb{F}_{4}+u \mathbb{F}_{4}}:\left(\mathbb{F}_{4}+u \mathbb{F}_{4}\right)^{n} \rightarrow \mathbb{F}_{4}^{2 n} \\
& a+b u \mapsto(b, a+b), a, b \in \mathbb{F}_{4}^{n}
\end{aligned}
$$

These maps preserve orthogonality in the corresponding alphabets. Moreover, the binary images $\varphi_{\mathbb{F}_{2}+u \mathbb{F}_{2}} \circ \psi_{\mathbb{F}_{4}+u \mathbb{F}_{4}}(C)$ and $\psi_{\mathbb{F}_{4}} \circ \varphi_{\mathbb{F}_{4}+u \mathbb{F}_{4}}(C)$ are equivalent. The Lee weight of an element is defined to be the Hamming weight of its binary image under the Gray map. we have the following result from [28].

Proposition 2.5. ([28]) Let $C$ be a code over $\mathbb{F}_{4}+u \mathbb{F}_{4}$. If $C$ is self-orthogonal, so are $\psi_{\mathbb{F}_{4}+u \mathbb{F}_{4}}(C)$ and $\varphi_{\mathbb{F}_{4}+u \mathbb{F}_{4}}(C)$. The code $C$ is Type $I$ (resp. Type II) over $\mathbb{F}_{4}+u \mathbb{F}_{4}$ if and only if $\varphi_{\mathbb{F}_{4}+u \mathbb{F}_{4}}(C)$ is a Type I (resp. Type II) $\mathbb{F}_{4}$-code, if and only if $\psi_{\mathbb{F}_{4}+u \mathbb{F}_{4}}(C)$ is a Type $I$ (resp. Type II) $\mathbb{F}_{2}+u \mathbb{F}_{2}$-code. Furthermore, the minimum Lee weight of $C$ is the same as the minimum Lee weight of $\psi_{\mathbb{F}_{4}+u \mathbb{F}_{4}}(C)$ and $\varphi_{\mathbb{F}_{4}+u \mathbb{F}_{4}}(C)$. 
Corollary 2.6. Suppose that $C$ is a self-dual code over $\mathbb{F}_{4}+u \mathbb{F}_{4}$ of length $n$ and minimum Lee distance $d$. Then $\varphi_{\mathbb{F}_{2}+u \mathbb{F}_{2}} \circ \psi_{\mathbb{F}_{4}+u \mathbb{F}_{4}}(C)$ is a binary $[4 n, 2 n, d]$ self-dual code. Moreover, $C$ and $\varphi_{\mathbb{F}_{2}+u \mathbb{F}_{2}} \circ \psi_{\mathbb{F}_{4}+u \mathbb{F}_{4}}(C)$ have the same weight enumerator. If $C$ is Type $I$ (Type II), then so is $\varphi_{\mathbb{F}_{2}+u \mathbb{F}_{2}} \circ \psi_{\mathbb{F}_{4}+u \mathbb{F}_{4}}(C)$.

\subsection{Certain Matrices and Group Rings}

In this section, we shall introduce group rings and a special isomorphism between the group ring a certain subring of $n \times n$ matrices over a ring. Before we introduce group rings, we need to define a block circulant. For further details on circulant matrices see [5].

Definition 2. A block circulant matrix over a ring $R$ is a square $k n \times k n$ matrix, which takes the form

$$
\operatorname{CIRC}\left(A_{1}, A_{2}, \ldots, A_{n}\right)=\left(\begin{array}{ccccc}
A_{1} & A_{2} & A_{3} & \ldots & A_{n} \\
A_{n} & A_{1} & A_{2} & \ldots & A_{n-1} \\
A_{n-1} & A_{n} & A_{1} & \ldots & A_{n-2} \\
\vdots & \vdots & \vdots & \ddots & \vdots \\
A_{2} & A_{3} & A_{4} & \ldots & A_{1}
\end{array}\right)
$$

where each $A_{i}$ is a $k \times k$ matrix over $R$.

Let $G$ be a finite group or order $n$, then the group ring $R G$ consists of $\sum_{i=1}^{n} \alpha_{i} g_{i}, \alpha_{i} \in R$, $g_{i} \in G$. Addition in the group ring is done by coordinate addition, namely

$$
\sum_{i=1}^{n} \alpha_{i} g_{i}+\sum_{i=1}^{n} \beta_{i} g_{i}=\sum_{i=1}^{n}\left(\alpha_{i}+\beta_{i}\right) g_{i} .
$$

The product of two elements in a group ring is given by

$$
\left(\sum_{i=1}^{n} \alpha_{i} g_{i}\right)\left(\sum_{j=1}^{n} \beta_{j} g_{j}\right)=\sum_{i, j} \alpha_{i} \beta_{j} g_{i} g_{j} .
$$

It follows that the coefficient of $g_{i}$ in the product is $\sum_{g_{i} g_{j}=g_{k}} \alpha_{i} \beta_{j}$.

We shall restrict ourselves to finite groups since we are using these to construct codes of finite lengths, however group rings can be defined for infinite groups as well. Throughout, we shall use $e_{G}$ to denote the identity element of any group $G$.

The following construction of a matrix was first given by Hurley in [22]. Let $R$ be a finite commutative Frobenius ring of characteristic 2 and let $G=\left\{g_{1}, g_{2}, \ldots, g_{n}\right\}$ be a group of order $n$. Let $v=\alpha_{g_{1}} g_{1}+\alpha_{g_{2}} g_{2}+\cdots+\alpha_{g_{n}} g_{n} \in R G$. Define the matrix $\sigma(v) \in M_{n}(R)$ to be

$$
\sigma(v)=\left(\begin{array}{ccccc}
\alpha_{g_{1}^{-1} g_{1}} & \alpha_{g_{1}^{-1} g_{2}} & \alpha_{g_{1}^{-1} g_{3}} & \ldots & \alpha_{g_{1}^{-1} g_{n}} \\
\alpha_{g_{2}^{-1} g_{1}} & \alpha_{g_{2}^{-1} g_{2}} & \alpha_{g_{2}^{-1} g_{3}} & \ldots & \alpha_{g_{2}^{-1} g_{n}} \\
\vdots & \vdots & \vdots & \vdots & \vdots \\
\alpha_{g_{n}^{-1} g_{1}} & \alpha_{g_{n}^{-1} g_{2}} & \alpha_{g_{n}^{-1} g_{3}} & \ldots & \alpha_{g_{n}^{-1} g_{n}}
\end{array}\right) .
$$


The elements $g_{1}^{-1}, g_{2}^{-1}, \ldots, g_{n}^{-1}$ are simply the elements of the group $G$ in a specific order. The construction in the next section depends on group rings where the order of the group is $4 p$ and $p$ is odd. In this paper, we consider $R C_{4 p}, R D_{4 p}$ and $R A_{4}$ where $C_{4 p}$ is the cyclic group of order $4 p, D_{4 p}$ is the dihedral group of order $4 p$ and $A_{4}$ is the alternating group on 4 elements. We shall now describe $\sigma(v)$ for each case.

1. Let $C_{4 p}=\left\langle x \mid x^{4 p}=1\right\rangle$ and

$$
v=\sum_{i=0}^{p-1} \sum_{j=0}^{3} \alpha_{i+p j+1} x^{4 i+j} \in R C_{4 p}
$$

then,

$$
\sigma(v)=\left(\begin{array}{cccc}
A_{1} & A_{2} & A_{3} & A_{4} \\
A_{4}^{\prime} & A_{1} & A_{2} & A_{3} \\
A_{3}^{\prime} & A_{4}^{\prime} & A_{1} & A_{2} \\
A_{2}^{\prime} & A_{3}^{\prime} & A_{4}^{\prime} & A_{1}
\end{array}\right)
$$

where $A_{i}=\operatorname{circ}\left(\alpha_{(i-1) p+1}, \ldots, \alpha_{i p}\right)$ and $A_{i}^{\prime}=\operatorname{circ}\left(\alpha_{i p}, \alpha_{(i-1) p+1}, \ldots, \alpha_{i p-1}\right)$.

2. Let $D_{4 p}=\left\langle x, y \mid x^{2 p}=y^{2}=1, x^{y}=x^{-1}\right\rangle$ and

$$
v=\sum_{i=0}^{p-1} x^{2 i}\left(\alpha_{i+1}+\alpha_{i+p+1} x+\alpha_{i+2 p+1} y+\alpha_{i+3 p+1} x y\right) \in R D_{4 p}
$$

then,

$$
\sigma(v)=\left(\begin{array}{cccc}
A_{1} & A_{2} & A_{3} & A_{4} \\
A_{2}^{\prime} & A_{1} & A_{4}^{\prime} & A_{3} \\
A_{3}^{T} & A_{4}^{\prime T} & A_{1}^{T} & A_{2}^{\prime T} \\
A_{4}^{T} & A_{3}^{T} & A_{2}^{T} & A_{1}^{T}
\end{array}\right)
$$

where $A_{i}=\operatorname{circ}\left(\alpha_{(i-1) p+1}, \ldots, \alpha_{i p}\right)$ and $A_{i}^{\prime}=\operatorname{circ}\left(\alpha_{i p}, \alpha_{(i-1) p+1}, \ldots, \alpha_{i p-1}\right)$.

3. Let $a=(1,2)(3,4), b=(1,3)(2,4)$ and $c=(1,2,3)$ where $a, b, c \in A_{4}$. If

$$
v=\sum_{i=0}^{2}\left(\alpha_{i+1}+\alpha_{i+4} a+\alpha_{i+7} b+\alpha_{i+10} a b\right) c^{i} \in R A_{4}
$$

then

$$
\sigma(v)=\left(\begin{array}{cccccccccccc}
\alpha_{1} & \alpha_{2} & \alpha_{3} & \alpha_{4} & \alpha_{5} & \alpha_{6} & \alpha_{7} & \alpha_{8} & \alpha_{9} & \alpha_{10} & \alpha_{11} & \alpha_{12} \\
\alpha_{3} & \alpha_{1} & \alpha_{2} & \alpha_{12} & \alpha_{10} & \alpha_{11} & \alpha_{6} & \alpha_{4} & \alpha_{5} & \alpha_{9} & \alpha_{7} & \alpha_{8} \\
\alpha_{2} & \alpha_{3} & \alpha_{1} & \alpha_{8} & \alpha_{9} & \alpha_{7} & \alpha_{11} & \alpha_{12} & \alpha_{10} & \alpha_{5} & \alpha_{6} & \alpha_{4} \\
\alpha_{4} & \alpha_{5} & \alpha_{6} & \alpha_{1} & \alpha_{2} & \alpha_{3} & \alpha_{10} & \alpha_{11} & \alpha_{12} & \alpha_{7} & \alpha_{8} & \alpha_{9} \\
\alpha_{12} & \alpha_{10} & \alpha_{11} & \alpha_{3} & \alpha_{1} & \alpha_{2} & \alpha_{9} & \alpha_{7} & \alpha_{8} & \alpha_{6} & \alpha_{4} & \alpha_{5} \\
\alpha_{8} & \alpha_{9} & \alpha_{7} & \alpha_{2} & \alpha_{3} & \alpha_{1} & \alpha_{5} & \alpha_{6} & \alpha_{4} & \alpha_{11} & \alpha_{12} & \alpha_{10} \\
\alpha_{7} & \alpha_{8} & \alpha_{9} & \alpha_{10} & \alpha_{11} & \alpha_{12} & \alpha_{1} & \alpha_{2} & \alpha_{3} & \alpha_{4} & \alpha_{5} & \alpha_{6} \\
\alpha_{6} & \alpha_{4} & \alpha_{5} & \alpha_{9} & \alpha_{7} & \alpha_{8} & \alpha_{3} & \alpha_{1} & \alpha_{2} & \alpha_{12} & \alpha_{10} & \alpha_{11} \\
\alpha_{11} & \alpha_{12} & \alpha_{10} & \alpha_{5} & \alpha_{6} & \alpha_{4} & \alpha_{2} & \alpha_{3} & \alpha_{1} & \alpha_{8} & \alpha_{9} & \alpha_{7} \\
\alpha_{10} & \alpha_{11} & \alpha_{12} & \alpha_{7} & \alpha_{8} & \alpha_{9} & \alpha_{4} & \alpha_{5} & \alpha_{6} & \alpha_{1} & \alpha_{2} & \alpha_{3} \\
\alpha_{9} & \alpha_{7} & \alpha_{8} & \alpha_{6} & \alpha_{4} & \alpha_{5} & \alpha_{12} & \alpha_{10} & \alpha_{11} & \alpha_{3} & \alpha_{1} & \alpha_{2} \\
\alpha_{5} & \alpha_{6} & \alpha_{4} & \alpha_{11} & \alpha_{12} & \alpha_{10} & \alpha_{8} & \alpha_{9} & \alpha_{7} & \alpha_{2} & \alpha_{3} & \alpha_{1}
\end{array}\right) .
$$




\subsection{Motivation}

In this subsection, we shall describe the motivation for the construction given in the next section. We begin with a lemma.

Lemma 2.7. Let $R$ be a finite commutative Frobenius ring of characteristic 2. Let $C$ be the code generated by a matrix $M$ of the form

$$
\left(\begin{array}{cc}
I_{k} & B \\
B^{T} & I_{k}
\end{array}\right),
$$

where $B$ is a $k$ by $k$ matrix. If the free rank of $C$ is $k$ then $C$ is self-dual.

Proof. Let the code $D$ be defined by $D=\left\langle\left(I_{k} \mid B\right)\right\rangle$ and $D^{\prime}=\left\langle\left(B^{T} \mid I_{k}\right)\right\rangle$. The inner-product of the $i$-th row of $\left(I_{k} \mid B\right)$ and the $j$-th row of $\left(B^{T} \mid I_{k}\right)$ is $B_{i, j}+B_{j, i}^{T}=B_{i, j}+B_{i, j}=0$ since the characteristic is 2 . Therefore $D^{\prime}=D^{\perp}$ since $|D|\left|D^{\prime}\right|=|R|^{n}$.

Let the code $C$ be defined by $C=\left\langle D, D^{\perp}\right\rangle$. If $D \neq D^{\perp}$ then $|C|>|D|$. However, we are assuming that the free rank of $C$ is $k$. Hence $C=D=D^{\perp}$. This gives that $C$ is a self-dual code.

Let $D_{2 k}$ be the dihedral group of order $2 k$. We describe the group by $D_{2 k}=\langle a, b| a^{2}=$ $\left.b^{k}=1, a b=b^{-1} a\right\rangle$.

We shall take a different ordering of the elements of the group than was given in [8]. Here the ordering of the elements for the map $\sigma$ is $1, b^{k-1}, b^{k-2}, \ldots, b^{2}, b^{1}, a, a b, a b^{2}, \ldots, a b^{k-1}$.

Let $v=\sum \alpha_{a^{i} b^{j}} a^{i} b^{j}$. With this ordering for the first row but multiplying in the order $1, b, b^{2}, \ldots, b^{k-1}, a, a b, a b^{2}, \ldots, a b^{k-1}$ on the left, the matrix $\sigma(v)$ is of the form:

$$
\left(\begin{array}{cccccccccc}
\alpha_{1} & \alpha_{b^{k-1}} & \alpha_{b^{k-2}} & \ldots & \alpha_{b} & \alpha_{a} & \alpha_{a b} & \alpha_{a b^{2}} & \ldots & \alpha_{a b^{k-1}} \\
\alpha_{b} & \alpha_{1} & \alpha_{b^{k-1}} & \ldots & \alpha_{b^{2}} & \alpha_{a b^{k-1}} & \alpha_{a} & \alpha_{a b} & \ldots & \alpha_{a b^{k-2}} \\
\vdots & \vdots & \vdots & \vdots & \vdots & \vdots & \vdots & \vdots & \vdots & \vdots \\
\alpha_{b^{k-1}} & \alpha_{b^{k-2}} & \alpha_{b^{k-3}} & \ldots & \alpha_{1} & \alpha_{a b} & \alpha_{a b^{2}} & \alpha_{a b^{3}} & \ldots & \alpha_{a} \\
\alpha_{a} & \alpha_{a b^{k-1}} & \alpha_{a b^{k-2}} & \ldots & \alpha_{a b} & \alpha_{1} & \alpha_{b} & \alpha_{b^{2}} & \ldots & \alpha_{b^{k-1}} \\
\alpha_{a b} & \alpha_{a} & \alpha_{a b^{k-1}} & \ldots & \alpha_{a b^{2}} & \alpha_{b^{k-1}} & \alpha_{1} & \alpha_{b} & \ldots & \alpha_{b^{k-2}} \\
\vdots & \vdots & \vdots & \vdots & \vdots & \vdots & \vdots & \vdots & \vdots & \vdots \\
\alpha_{a b^{k-1}} & \alpha_{a b^{k-2}} & \alpha_{a b^{k-3}} & \ldots & \alpha_{a b^{k-2}} & \alpha_{b} & \alpha_{b^{2}} & \alpha_{b^{3}} & \ldots & \alpha_{1}
\end{array}\right)
$$

This gives that $\sigma(v)$ is of the form:

$$
\left(\begin{array}{cc}
A & B \\
B^{T} & A^{T}
\end{array}\right)
$$

where $A$ and $B$ are circulant matrices. 
Theorem 2.8. Let $R$ be a finite commutative Frobenius ring of characteristic 2 and let $v \in R D_{2 k}$ with $v=\sum \alpha_{a^{i} b^{j}} a^{i} b^{j}$ where only $\alpha_{a^{0} b}$ is 1 and $\alpha_{a^{0} b^{i}}$ is 0 for $i \neq 1$. Let $C_{v}=\langle\sigma(v)\rangle$. If $C_{v}$ has free rank $k$, then $C_{v}$ is a self-dual code.

Proof. Since $\alpha_{a^{0} v}$ is 1 and $\alpha_{a^{0} b^{i}}$ is 0 for $i \neq 1$, the generator matrix of $C_{v}$ is of the form:

$$
\left(\begin{array}{cc}
I_{k} & B \\
B^{T} & I_{k}
\end{array}\right)
$$

Then, by Lemma 2.7, we have the result.

Corollary 2.9. Let $C$ be a self-dual code over a finite commutative Frobenius ring of characteristic 2 of length $2 k$ generated by $\left(I_{k} \mid A\right)$ where $A$ is a circulant matrix. Then $C$ is an ideal in $R D_{2 k}$ and $D_{2 k}$ is a subgroup of the automorphism group of $C$.

Proof. The orthogonal of the code generated by $\left(I_{k} \mid A\right)$ is the code generated by $\left(A^{T} \mid I_{k}\right)$.

Since $C$ is self-dual then the code generated by $\left(\begin{array}{cc}I_{k} & A \\ A^{T} & I_{k}\end{array}\right)$ where $A$ is a circulant matrix is the self-dual code $C$. Therefore $C=\langle\sigma(v)\rangle$ for some $v \in R D_{2 k}$.

The fact that the code has $D_{2 k}$ as a subgroup of its automorphism group follows from Corollary 2.2 in [8].

The implication of this corollary is that if you are simply searching for self-dual codes generated by $\left(I_{k} \mid A\right)$ where $A$ is a circulant matrix then you will only be finding codes with a fairly significant restriction on their automorphism group. Additionally, it was shown in [8] that if $A$ is a reverse circulant matrix that it will correspond to an ideal in the same group algebra with a different ordering of the elements. This means that, in either case, you will only be finding codes which correspond to ideals in in the group ring $R D_{2 k}$. One implication of this is that you can only find self-dual codes where $2 k$ divides the order of the automorphism group. Therefore, if we wish to find all interesting self-dual codes of a given length (for example the extremal codes) then we need to expand the structure of matrices that we use to construct the codes. This is precisely what we do in this paper. We give new structures for matrices to obtain codes that were previously missed by other constructions.

\section{Construction}

Let $v \in R G$ where $R$ is a finite commutative ring and $G$ is a finite group. If $v=\sum_{g} a_{g} g$ then $v^{*}=\sum_{g} a_{g} g^{-1}$. We say that $v$ is a unitary unit in $R G$ if $v v^{*}=1$.

Let $v \in R G$ where $R$ is a finite commutative Frobenius ring of characteristic 2 and $G$ is a finite group of order $4 p$ where $p$ is odd. Let $a_{i} \in R$ and $\mathbf{a}_{\mathbf{i}+4}=\left(a_{i+4}, \ldots, a_{i+4}\right) \in R^{p}$ for $1 \leq i \leq 4$. Define the following matrix: 


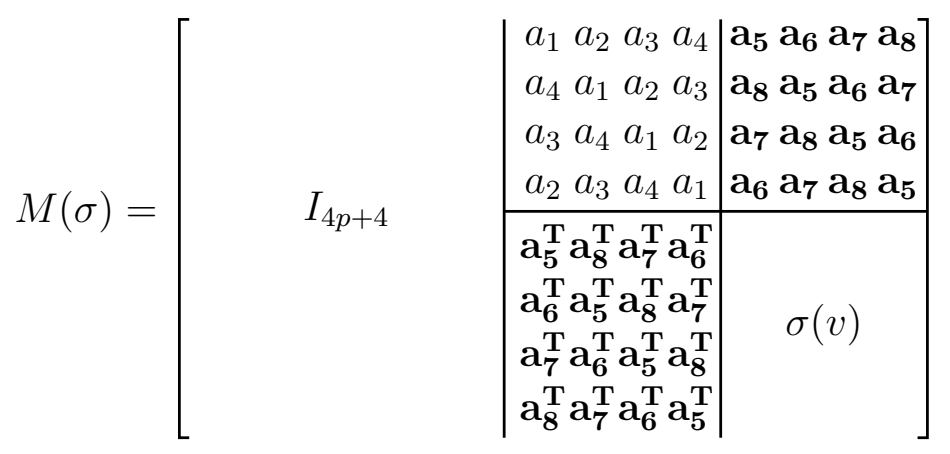

Let $C_{\sigma}$ be a code that is generated by the matrix $M(\sigma)$. Then, the code $C_{\sigma}$ has length $8 p+8$. We want to investigate when this construction yields self-dual codes. Consequently, we will now consider $M(\sigma) M(\sigma)^{T}$.

Let $A=\operatorname{circ}\left(a_{1}, a_{2}, a_{3}, a_{4}\right)$ and $B=C I R C\left(\mathbf{a}_{\mathbf{5}}, \mathbf{a}_{\mathbf{6}}, \mathbf{a}_{\mathbf{7}}, \mathbf{a}_{\mathbf{8}}\right)$. Then

$$
\begin{aligned}
M(\sigma) M(\sigma)^{T} & =I+\left(\begin{array}{cc}
A & B \\
B^{T} & \sigma(v)
\end{array}\right)\left(\begin{array}{cc}
A^{T} & B \\
B^{T} & \sigma(v)^{T}
\end{array}\right) \\
& =I+\left(\begin{array}{cc}
A & B \\
B^{T} & \sigma(v)
\end{array}\right)\left(\begin{array}{cc}
A^{T} & B \\
B^{T} & \sigma\left(v^{*}\right)
\end{array}\right) \\
& =\left(\begin{array}{cc}
A A^{T}+B B^{T}+I & A B+B \sigma\left(v^{*}\right) \\
B^{T} A^{T}+\sigma(v) B^{T} & B^{T} B+\sigma\left(v v^{*}\right)+I
\end{array}\right) .
\end{aligned}
$$

Now, $A A^{T}=\operatorname{circ}\left(\sum_{i=1}^{4} a_{i}^{2},\left(a_{1}+a_{3}\right)\left(a_{2}+a_{4}\right), 0,\left(a_{1}+a_{3}\right)\left(a_{2}+a_{4}\right)\right)$ and

$$
\begin{aligned}
B B^{T} & =\operatorname{circ}\left(p \sum_{i=1}^{4} a_{i+4}^{2}, p\left(a_{5}+a_{7}\right)\left(a_{6}+a_{8}\right), 0, p\left(a_{5}+a_{7}\right)\left(a_{6}+a_{8}\right)\right) \\
& =\operatorname{circ}\left(\sum_{i=1}^{4} a_{i+4}^{2},\left(a_{5}+a_{7}\right)\left(a_{6}+a_{8}\right), 0,\left(a_{5}+a_{7}\right)\left(a_{6}+a_{8}\right)\right) .
\end{aligned}
$$

Therefore

$$
A A^{T}+B B^{T}+I=\operatorname{circ}\left(1+\sum_{i=1}^{8} a_{i}^{2}, \gamma, 0, \gamma\right)
$$

where $\gamma=\left(a_{1}+a_{3}\right)\left(a_{2}+a_{4}\right)+\left(a_{5}+a_{7}\right)\left(a_{6}+a_{8}\right)$. Additionally,

$$
B^{T} B=C I R C\left(\sum_{i=1}^{4} a_{i+4}(\operatorname{circ}(\underbrace{1, \ldots, 1}_{p \text {-times }})), \delta(\operatorname{circ}(\underbrace{1, \ldots, 1}_{p \text {-times }})), 0, \delta(\operatorname{circ}(\underbrace{1, \ldots, 1}_{p \text {-times }}))\right)
$$

where $\delta=\left(a_{5}+a_{7}\right)\left(a_{6}+a_{8}\right)$.

We will now provide conditions when the above construction produces self-dual codes. We also provide a connection (when using this construction) between self-dual codes and units and non-units in a group ring. 
Theorem 3.1. Let $R$ be a finite commutative Frobenius ring of characteristic 2 and let $G$ be a finite group of order $4 p$ where $p$ is odd. If $A A^{T}+B B^{T}+I=0, A B+B \sigma\left(v^{*}\right)=0$ and $B^{T} B+\sigma\left(v v^{*}\right)+I=0$ then $C_{\sigma}$ is a self-dual code of length $8 p+8$.

Proof. Clearly, $C_{\sigma}$ has free rank $4 p+4$ as the left hand side of the generator matrix is the $4 p+4$ by $4 p+4$ identity matrix. If $A A^{T}+B B^{T}+I=0, A B+B \sigma\left(v^{*}\right)=0$ and $B^{T} B+\sigma\left(v v^{*}\right)+I=0$ then $C_{\sigma}$ is self-orthogonal and $C_{\sigma}$ is self-dual.

Corollary 3.2. Let $R$ be a finite commutative Frobenius ring of characteristic 2 , and let $G$ be a finite group of order $4 p$ where $p$ is odd. Let $v \in R G$ be a unitary unit. If $a_{5}=a_{7}$, $a_{6}=a_{8}, a_{1}=a_{3}$ and $a_{2}+a_{4}=1$ or $a_{5}=a_{7}, a_{6}=a_{8}, a_{1}+a_{3}=1$ and $a_{2}=a_{4}$ then $C_{\sigma}$ is $a$ self-dual code of length $8 p+8$.

Proof. If $a_{5}=a_{7}, a_{6}=a_{8}, a_{1}=a_{3}$ and $a_{2}+a_{4}=1$ or $a_{5}=a_{7}, a_{6}=a_{8}, a_{1}+a_{3}=1$ and $a_{2}=a_{4}$ then $A A^{T}+B B^{T}+I=0$ and $B^{T} B+\sigma\left(v v^{*}\right)+I=\sigma\left(v v^{*}\right)+I=2 I=0$ since $I$ is unitary. Therefore $C_{\sigma}$ is self-dual.

Corollary 3.3. Let $R$ be a finite commutative Frobenius ring of characteristic 2, and let $G$ be a finite group of order $4 p$ where $p$ is odd. Let $C_{\sigma}$ be self-dual. If $\sum_{i=1}^{4} a_{i+4}=1$, then $v \in R G$ is a non-unit.

Proof. If $C_{\sigma}$ is self-dual, clearly $\sigma\left(v v^{*}\right)=B^{T} B+I$. If $\sum_{i=1}^{4} a_{i+4}=1$, then $I+B^{T} B=$ $C I R C\left(A_{1}, A_{2}, 0, A_{2}\right)$ where $A_{1}=\operatorname{circ}(0, \underbrace{1, \ldots, 1}_{(p-1) \text {-times }})$ and $A_{2}=\operatorname{circ}(\underbrace{\delta, \ldots, \delta}_{p-\text { times }})$. Let $C=$ $C I R C\left(A_{1}, A_{2}, 0, A_{2}\right)$. For each $2 \leq i \leq 4 p$, add the $i$-th row of $C$ to the first row of $C$. Then the first row becomes

$$
\underbrace{((p-1)+2 p \delta,(p-1)+2 p \delta, \ldots(p-1)+2 p \delta}_{4 p \text {-times }})=\underbrace{(0,0, \ldots, 0)}_{4 p-\text { times }} .
$$

Since $p$ is odd and the ring has characteristic 2 , then $\operatorname{det}\left(\operatorname{CIRC}\left(A_{1}, A_{2}, 0, A_{2}\right)\right)=0$. Therefore, $\operatorname{det}\left(I+B^{T} B\right)=0$ and $v v^{*}$ is a non-unit by Corollary 3 in [22]. Therefore, $v \in R G$ is a non-unit.

We can use the structure of the family of rings $R_{s}$ to get an infinite number of binary self-dual codes from a single matrix $M$ satisfying the conditions of Theorem 3.1.

Theorem 3.4. If $M$ is a matrix satisfying the conditions in Theorem 3.1 over $R_{k}$, then $M$ generates a self-dual code over $R_{s}$ for all $s \geq k$.

Proof. If $k \leq s$ then the ring $R_{k}$ is a subring of the ring $R_{s}$. The matrix $M$ has free rank $4 p+4$ over any ring where it is defined and if $\mathbf{v}$ and $\mathbf{w}$ are orthogonal over $R_{k}$ then they are orthogonal over $R_{s}$ as well since $R_{k}$ is a subring of $R_{s}$. Therefore, the code generated by $M$ over $R_{s}$ is a self-dual code of length $8 p+8$. 
This leads immediately to the following corollary.

Corollary 3.5. Let $M$ be a matrix satisfying the conditions in Theorem 3.1 over $R_{k}$. Let $C_{s}$ be the code generated by $M$ over $R_{s}$. Then $\phi_{s}\left(C_{s}\right)$ is a binary self-dual code of length $2^{s}(8 p+8)$.

Similarly, we have the following.

Theorem 3.6. If $M$ is a matrix satisfying the conditions in Theorem 3.1 over $R_{1}$, then $M$ generates a self-dual code over $\mathbb{F}_{4}+u \mathbb{F}_{4}$.

Proof. The ring $R_{1}$ is a subring of the ring $\mathbb{F}_{4}+u \mathbb{F}_{4}$. Therefore the proof follows exactly as the proof of Theorem 3.4.

This leads naturally to the following corollary using the result in Corollary 2.6.

Corollary 3.7. Let $M$ be a matrix satisfying the conditions in Theorem 3.1 over $R_{1}$. Let $C$ be the code generated by $M$ over $\mathbb{F}_{4}+u \mathbb{F}_{4}$. Then $\left(\phi_{\mathbb{F}_{2}+u \mathbb{F}_{2}} \circ \psi_{\mathbb{F}_{4}+u \mathbb{F}_{4}}\right)(C)$ is a binary self-dual code of length $4(8 p+8)$.

\section{Results}

In this section, we will present the results obtained using this construction to construct self-dual codes for certain groups of order 4, 12 and 20.

\subsection{Constructions coming from a group of order 4}

Here we present the results for the above construction using $G=C_{4}$. We construct self-dual codes of length 16,32 and 64 by considering this construction over $\mathbb{F}_{2}, \mathbb{F}_{4}$ and $\mathbb{F}_{4}+u \mathbb{F}_{4}$. Finally, we construct new extremal self-dual codes of length 68 by extending certain extremal self-dual codes of length 64 .

The possible weight enumerators for a self-dual Type I $[64,32,12]$-code is given in $[4,10]$ as:

$$
\begin{aligned}
& W_{64,1}=1+(1312+16 \beta) y^{12}+(22016-64 \beta) y^{14}+\cdots, 14 \leq \beta \leq 284 \\
& W_{64,2}=1+(1312+16 \beta) y^{12}+(23040-64 \beta) y^{14}+\cdots, 0 \leq \beta \leq 277
\end{aligned}
$$

With the most updated information, the existence of codes is known for $\beta=14,18,22,25$, $29,32,35,36,39,44,46,53,59,60,64$ and 74 in $W_{64,1}$ and for $\beta=0,1,2,4,5,6,8,9,10$, $12,13,14,16, \ldots, 25,28,19,30,32,33,34,36,37,38,40,41,42,44,45,48,50,51,52,56$, $58,64,72,80,88,96,104,108,112,114,118,120$ and 184 in $W_{64,2}$. 
Table 1: Self-dual code over $\mathbb{F}_{4}+u \mathbb{F}_{4}$ of length 64 from $C_{4}$ where each code is of type $W_{64,2}$

\begin{tabular}{|c|c|c|c|c|c|c|c|c|c|}
\hline$C_{i}$ & $\left(a_{1}, \ldots, a_{8}\right)$ & $\left(\alpha_{1}, \ldots, \alpha_{4}\right)$ & $|A u t(C)|$ & $\beta$ & $C_{i}$ & $\left(a_{1}, \ldots, a_{8}\right)$ & $\left(\alpha_{1}, \ldots, \alpha_{4}\right)$ & $|A u t(C)|$ & $\beta$ \\
\hline 1 & $(0,9,6,4,2,4,3,5)$ & $(9, D, F, 0)$ & $2^{4}$ & 0 & 2 & $(0,4,9, F, 1,1,6,7)$ & $(0, F, 9,4)$ & $2^{5}$ & 0 \\
\hline 3 & $(2,2,1,1,0,9,6,4)$ & $(2,1,1,2)$ & $2^{6}$ & 0 & 4 & $(0,9,6, E, A, 4,3, F)$ & $(1, D, 7, A)$ & $2^{3}$ & 2 \\
\hline 5 & $(2,8,4,5,6,6, F, F)$ & $(4,3,2, E)$ & $2^{4}$ & 2 & 6 & $(0,9,6, E, 2,4,1,5)$ & $(5,1,3,6)$ & $2^{3}$ & 4 \\
\hline 7 & $(2,4,1, F, 9,9, E, D)$ & $(2, F, 1,4)$ & $2^{4}$ & 4 & 8 & $(A, A, 9,9,0,9, E, E)$ & $(A, 9,9, A)$ & $2^{5}$ & 4 \\
\hline 9 & $(2,9,4,6,0,4,1, D)$ & $(7,1,1, E)$ & $2^{3}$ & 6 & 10 & $(0, A, 6,7, A, A, 9,9)$ & $(6,1,0, C)$ & $2^{4}$ & 6 \\
\hline 11 & $(0,1,6,6,8,6, B, 7)$ & $(9, D, F, A)$ & $2^{3}$ & 8 & 12 & $(2,4,9, F, 1,9,6,7)$ & $(2, F, 9,4)$ & $2^{4}$ & 8 \\
\hline 13 & $(0,0,2,6,1,7, E, D)$ & $(0,6,2,0)$ & $2^{5}$ & 8 & 14 & $(2,2,4,7,2,4,3, F)$ & $(4,3,2,6)$ & $2^{3}$ & 10 \\
\hline 15 & $(A, 0,4,7,6,6, D, D)$ & $(6, B, 8, C)$ & $2^{4}$ & 10 & 16 & $(0,9,6, E, 8,4,9,7)$ & $(5,1,3,6)$ & $2^{3}$ & 12 \\
\hline 17 & $(0,4,1, D, 9,3, E, 7)$ & $(0, D, 1,4)$ & $2^{4}$ & 12 & 18 & $(2,2,4,5,4, C, 5, F)$ & $(9, C, F, B)$ & $2^{5}$ & 12 \\
\hline 19 & $(0, A, 6,7,2,4,1,7)$ & $(8, F, E, 2)$ & $2^{3}$ & 14 & 20 & $(A, 8,4,5,6, E, F, 5)$ & $(6,1,8, C)$ & $2^{4}$ & 14 \\
\hline 21 & $(0,1,6,4,8,4,9, F)$ & $(3, D, 5,8)$ & $2^{3}$ & 16 & 22 & $(2,4,9,5,1,9, C, D)$ & $(2,5,9,4)$ & $2^{4}$ & 16 \\
\hline 23 & $(A, A, 9,9,2,1, C, 6)$ & $(A, 9,9, A)$ & $2^{5}$ & 16 & 24 & $(0,1,6, C, 2,6,3,7)$ & $(D, 1, B, C)$ & $2^{3}$ & 18 \\
\hline 25 & $(8, A, 6,7,4, C, D, 7)$ & $(4,3, A, E)$ & $2^{4}$ & 18 & 26 & $(2,4,1,5,9,9,4,7)$ & $(2,5,1,4)$ & $2^{4}$ & 20 \\
\hline 27 & $(2,2,9,9,1, B, 6,5)$ & $(2,9,9,2)$ & $2^{5}$ & 20 & 28 & $(0,1,4, C, 2,6,1, F)$ & $(9, F, D, 2)$ & $2^{3}$ & 22 \\
\hline 29 & $(0,8,6,5,6,6, D, D)$ & $(1,4,7,9)$ & $2^{4}$ & 22 & 30 & $(2,4,1, D, 9, B, 4, F)$ & $(2, D, 1,4)$ & $2^{4}$ & 24 \\
\hline 31 & $(2,2,9,9,1,9, C, F)$ & $(2,9,9,2)$ & $2^{5}$ & 24 & 32 & $(2,9,4,4,8,6,1, F)$ & $(F, 9,9,4)$ & $2^{3}$ & 26 \\
\hline
\end{tabular}

\subsubsection{New Codes of length 68 from $\left(\mathbb{F}_{4}+u \mathbb{F}_{4}\right) C_{4}$}

The possible weight enumerator of a self-dual $[68,34,12]_{2}$-code is in one of the following forms by $[2,21,9]$ :

$$
\begin{aligned}
& W_{68,1}=1+(442+4 \beta) y^{12}+(10864-8 \beta) y^{14}+\cdots, 104 \leq \beta \leq 1358 \\
& W_{68,2}=1+(442+4 \beta) y^{12}+(14960-8 \beta-256 \gamma) y^{14}+\cdots
\end{aligned}
$$

where $0 \leq \gamma \leq 9$. Recently, Yankov et. al. constructed the first examples of codes with a weight enumerator for $\gamma=7$ in $W_{68,2}$. In [9], more unknown $W_{68,2}$ codes were constructed. 
Table 2: Self-dual code over $\mathbb{F}_{4}+u \mathbb{F}_{4}$ of length 64 from $C_{4}$ where each code is of type $W_{64,2}$

\begin{tabular}{c|cccc||c|cccc}
\hline$C_{i}$ & $\left(a_{1}, \ldots, a_{8}\right)$ & $\left(\alpha_{1}, \ldots, \alpha_{4}\right)$ & $\mid$ Aut $(C) \mid$ & $\beta$ & $C_{i}$ & $\left(a_{1}, \ldots, a_{8}\right)$ & $\left(\alpha_{1}, \ldots, \alpha_{4}\right)$ & $|A u t(C)|$ & $\beta$ \\
\hline 33 & $(2,9,4,6,8,4,9, D)$ & $(B, 7, D, 8)$ & $2^{3}$ & 28 & 34 & $(2,6,9,7,1,1,4,7)$ & $(2,7,9,6)$ & $2^{4}$ & 28 \\
\hline 35 & $(2,2,4,5,6, E, 7, D)$ & $(9, C, F, B)$ & $2^{5}$ & 28 & 36 & $(0,0,6,7,4, C, D, 7)$ & $(1,4,7,3)$ & $2^{4}$ & 30 \\
\hline 37 & $(2,4,9,7,9,3,4, F)$ & $(2,7,9,4)$ & $2^{4}$ & 32 & 38 & $(A, 8,9,9,2,9,4, E)$ & $(A, 9,9,8)$ & $2^{5}$ & 32 \\
\hline 39 & $(A, 4,9, F, 1,1,4, F)$ & $(A, F, 9,4)$ & $2^{4}$ & 36 & 40 & $(0,0,9,9, A, 9,4, E)$ & $(0,9,9,0)$ & $2^{5}$ & 36 \\
\hline 41 & $(0, A, 2,6,1,5,6, F)$ & $(0,6,2, A)$ & $2^{5}$ & 40 & 42 & $(A, 8,4,5,4, E, F, 7)$ & $(3, E, D, 3)$ & $2^{4}$ & 44 \\
\hline 43 & $(0,0,4,7, A, 6,1,5)$ & $(0,7,4,0)$ & $2^{4} \cdot 3$ & 44 & 44 & $(8,8,1,1,0,9,6,4)$ & $(8,1,1,8)$ & $2^{5}$ & 48 \\
\hline 45 & $(A, A, 1,1,8,1, E, C)$ & $(A, 1,1, A)$ & $2^{5}$ & 52 & & & & \\
\hline
\end{tabular}

Together with these, the existence of the codes in $W_{68,2}$ is known for;

$\gamma=0, \beta=0,7,11,14,17,21,22,28,33,35,42,44, \ldots, 158,161,165$, $175,187,189,203,209,221,231,255,303$ or

$\beta \in\{2 m \mid m=17,20,102,110,119,136,165$ or $80 \leq m \leq 99\}$;

$\gamma=1, \beta=49,51,53,55,57,59, \ldots, 160$ or

$\beta \in\{2 m \mid m=25, \ldots, 29,81, \ldots, 90,92, \ldots, 96\}$;

$\gamma=2, \beta=65,69,71,73,75,77,79,81,159,206,208$ or $\beta \in\{2 m \mid 30 \leq m \leq 68,70 \leq m \leq 100\}$ or

$\beta \in\{2 m+1 \mid 41 \leq m \leq 69,71 \leq m \leq 77\}$;

$\gamma=3, \beta=84,95,97,101,103,105,107,109,113,115,117,119,121,123,125,127,129,131,133,135$, $137,139,141,143,145,147,149,151,153,155,159,161,193$ or

$\beta \in\{2 m \mid m=44,45,47, \ldots, 84,86, \ldots, 92,94,95,97,98,101,102\} ;$

$\gamma=4, \beta=129,141,145,157,161$ or

$\beta \in\{2 m \mid m=43,48,49,51,52,54,55,56,58,60, \ldots, 78,80,87,97,98\}$;

$\gamma=6$ with $\beta \in\{2 m \mid m=69,77,78,79,81,88\}$

$\gamma=7$ with $\beta \in\{7 m \mid m=14, \ldots, 39,42\}$.

Recall that the previously constructed codes of length 64 are codes over $\mathbb{F}_{4}+u \mathbb{F}_{4}$. In order to apply Theorem 2.4, it requires the codes to be over $\mathbb{F}_{2}+u \mathbb{F}_{2}$. Before considering extensions of these codes, we need to use the Gray map $\psi_{\mathbb{F}_{4}+u \mathbb{F}_{4}}$ to convert them to a code over $\mathbb{F}_{2}+u \mathbb{F}_{2}$. The following table details the new extremal self-dual codes of length 68 . For each new code constructed we note the original code of length 64 from the previous table, the unit $c \in \mathbb{F}_{2}+u \mathbb{F}_{2}$, the vector $X$ required to apply Theorem 2.4 and we replace $u+1$ with 3 to save space.

Self dual binary codes $C$ and $D$ of length $n$ are said to be neighbors if $\operatorname{dim}(C \cap D)=$ $n / 2-1$. In order to consider some neighbors of a code $C$ we pick a vector $x \in \mathbb{F}_{2}^{n}-C$ and 
Table 3: Extremal self-dual codes of length 68 from $C_{4}$ over $\mathbb{F}_{4}+u \mathbb{F}_{4}$.

\begin{tabular}{|c|c|c|c|c|c|c|}
\hline $\mathcal{C}_{68, i}$ & $\mathcal{C}_{i}$ & $c$ & $X$ & $\gamma$ & $\beta$ & $\left|A u t\left(\mathrm{C}_{68, i}\right)\right|$ \\
\hline 1 & 2 & 1 & (uи333033031110uи3u13u001u0133313) & 1 & 44 & 2 \\
\hline 2 & 2 & 1 & (131103u0uиu1011033uи033u0u0u3013) & 1 & 48 & 2 \\
\hline 3 & 2 & 1 & (3uи010u30uиu00013uи0u3131u0u1313) & 2 & 58 & 2 \\
\hline 4 & 2 & 1 & (3u0uu11313u0u01130130303u1uu00u3) & 3 & 86 & 2 \\
\hline 5 & 2 & 1 & (3u13u0u01uu013u10u13010131011uu0) & 4 & 100 & 2 \\
\hline 6 & 2 & $u+1$ & (0101u3u1uиu03и3и01301100u0101u30) & 4 & 114 & 2 \\
\hline 7 & 3 & 1 & (1u0133000010u313011133u01u331u33) & 3 & 87 & 2 \\
\hline 8 & 3 & 1 & (3100u303u331003u033u013u301u13u1) & 3 & 89 & 2 \\
\hline 9 & 4 & 1 & (313u030313u11u133130u1101u0u3uu1) & 3 & 99 & 2 \\
\hline 10 & 8 & 1 & (030uиu30010u0033131u1333иии03и11) & 3 & 92 & 2 \\
\hline 11 & 9 & 1 & $(00300 u 001001 u 00 u 330 u 33 u 3330111 u u)$ & 3 & 82 & 2 \\
\hline 12 & 10 & 1 & $(1 u 01000 u 1310 u 1 u 011 u 0111 u 1 u 103100)$ & 3 & 111 & 2 \\
\hline 13 & 21 & $u+1$ & $(u 3 u 11 u 113 u 1333 u 1 u 031333 u 1100131 u)$ & 4 & 139 & 2 \\
\hline 14 & 26 & $u+1$ & (ии0ии10uи11u3и0u03изи0иนии1u3331) & 4 & 143 & 2 \\
\hline 15 & 30 & 1 & (13u031u03330uи0uиu3313и330110310) & 4 & 149 & 2 \\
\hline 16 & 37 & 1 & (1000uи11u100uи0001uии310u0103u00) & 2 & 141 & 2 \\
\hline 17 & 40 & 1 & $(3300 u 30103010 u 111 u 311303 u 01303 u 0)$ & 4 & 158 & 2 \\
\hline 18 & 40 & $u+1$ & (u01101310u030110111110uи33и11uии) & 4 & 162 & 2 \\
\hline 19 & 40 & $u+1$ & (10031u301u01010ииииии311ии10u011) & 4 & 170 & 2 \\
\hline
\end{tabular}

let $D=\left\langle\langle x\rangle^{\perp} \cap C, x\right\rangle$. We use the standard form of the generator matrix of $C$, which lets us to fix first $n / 2$ entries of $x$ without loss of generality. We set the first 34 entries of $x$ to be 0 . We consider the neighbors of the binary images of the codes in Table 3 and obtain five new codes of length 68 which are listed in Table 4.

\subsection{Constructions coming from groups of order 12}

Here we present the results for the above construction using $G \in\left\{C_{12}, D_{12}, A_{4}\right\}$. We construct self-dual codes of length 32 and 64 by considering this construction over $\mathbb{F}_{2}$ and $\mathbb{F}_{2}+u \mathbb{F}_{2}$. Again, we construct new extremal self-dual codes of length 68 by extending certain extremal self-dual codes of length 64 .

\subsubsection{New Codes of length 68 from $R_{1} A_{4}$}

We now consider extensions of the previously constructed codes of length 64 (codes from $A_{4}$ over $\mathbb{F}_{2}+u \mathbb{F}_{2}$ ). The following table records newly constructed extremal self-dual codes of length 68. Again, we note the original code of length 64 from the previous table, the unit 
Table 4: New codes of length 68 with $\gamma=4$ as neighbors of codes in Table 3

\begin{tabular}{ccccc}
\hline $\mathcal{N}_{68, i}$ & $\mathcal{C}_{68, j}$ & $\left(x_{35}, x_{36}, \ldots, x_{68}\right)$ & $\beta$ & $\left|A u t\left(\mathcal{N}_{68, i}\right)\right|$ \\
\hline $\mathcal{N}_{68,1}$ & $\mathcal{C}_{68,19}$ & $(0010001001100111011111101110111001)$ & $\mathbf{1 6 6}$ & 2 \\
\hline $\mathcal{N}_{68,2}$ & $\mathcal{C}_{68,17}$ & $(0111100100010101001101011111011011)$ & $\mathbf{1 6 7}$ & 2 \\
\hline $\mathcal{N}_{68,3}$ & $\mathcal{C}_{68,17}$ & $(1110011101111010100011001100110110)$ & $\mathbf{1 6 8}$ & 2 \\
\hline $\mathcal{N}_{68,4}$ & $\mathcal{C}_{68,18}$ & $(1111101010110100110110110010101100)$ & $\mathbf{1 6 9}$ & 2 \\
\hline $\mathcal{N}_{68,5}$ & $\mathcal{C}_{68,19}$ & $(0011101101110100110111110011000000)$ & $\mathbf{1 7 1}$ & 2 \\
\hline
\end{tabular}

Table 5: Type II Extremal Self-dual code of length 64 from $D_{12}$ over $R_{1}$.

\begin{tabular}{ccc}
\hline$\left(a_{1}, \ldots, a_{8}\right)$ & $\left(\alpha_{1}, \ldots, \alpha_{12}\right)$ & $\mid$ Aut $(C) \mid$ \\
\hline$(u, 1,0,1, u, 0, u, 1)$ & $(u, 1,1, u, u+1,0, u, 1, u+1,1, u+1, u+1)$ & $2^{3} \cdot 3$ \\
\hline$(u, 1,0,1, u, 0, u, 1)$ & $(u, 1,1, u, u+1,0, u, 1, u+1, u+1, u+1,1)$ & $2^{3} \cdot 3$ \\
\hline
\end{tabular}

Table 6: Type II Extremal Self-dual code of length 64 from $A_{4}$ over $R_{1}$.

\begin{tabular}{ccc}
\hline$\left(a_{1}, \ldots, a_{8}\right)$ & $\left(\alpha_{1}, \ldots, \alpha_{12}\right)$ & $\mid$ Aut $(C) \mid$ \\
\hline$(1, u+1, u+1, u+1, u, 0, u, 1)$ & $(u, u, 1,0, u, 1,1, u, 0,1, u+1,1)$ & $2^{4} \cdot 3$ \\
\hline$(u+1,1,1,1, u, 0, u, 1)$ & $(0, u, 1, u, u, 1, u+1, u, 0, u+1, u+1,1)$ & $2^{4}$ \\
\hline
\end{tabular}

Table 7: Type I Extremal Self-dual code of length 64 from $A_{4}$ over $R_{1}$.

\begin{tabular}{ccccc}
\hline $\mathcal{D}_{i}$ & $\left(a_{1}, \ldots, a_{8}\right)$ & $\left(\alpha_{1}, \ldots, \alpha_{12}\right)$ & $|A u t(C)|$ & $W_{64,2}$ \\
\hline 1 & $(u+1,1,1,1, u, u, u, 1)$ & $(0, u, 1, u, u, 1, u+1, u, 0, u+1, u+1,1)$ & $2^{4} \cdot 3$ & $\beta=64$ \\
\hline 2 & $(1, u+1, u+1, u+1, u, u, u, 1)$ & $(0,0,1, u, 0,1,1,0, u, 1, u+1,1)$ & $2^{4} \cdot 3^{2}$ & $\beta=40$ \\
\hline 3 & $(u, 0,0,0, u, 1,1,1)$ & $(u, 1,1, u, u, 0,0,1,1, u+1,0, u+1)$ & $2^{3} \cdot 3$ & $\beta=52$ \\
\hline
\end{tabular}

$c \in \mathbb{F}_{2}+u \mathbb{F}_{2}$, the vector $X$ required to apply Theorem 2.4 and we replace $u+1$ with 3 to save space 
Table 8: Extremal Self-dual code of length 68 from $A_{4}$ over $R_{1}$.

\begin{tabular}{ccccccc}
\hline $\mathcal{D}_{68, i}$ & $\mathcal{D}_{i}$ & $c$ & $X$ & $\gamma$ & $\beta$ & $\mid$ Aut $\left(\mathrm{D}_{68, i}\right)$ \\
\hline 1 & 1 & $u+1$ & $(1 u u u u 1 u 13 u 1 u u 110113 u 0 u 33 u 301133 u)$ & $\mathbf{0}$ & $\mathbf{1 5 9}$ & 2 \\
\hline 2 & 1 & $u+1$ & $(03030000 u u 01 u 111 u 33 u 13 u 0 u 1 u 0 u u 11)$ & $\mathbf{0}$ & $\mathbf{1 6 3}$ & 2 \\
\hline 3 & 1 & 1 & $(31 u 03 u 003 u 1031 u 030 u u 0 u u 30110011 u)$ & $\mathbf{1}$ & $\mathbf{1 6 1}$ & 2 \\
\hline 4 & 1 & $u+1$ & $(300133130 u 110013303 u 0 u 1 u u 1 u 30013)$ & $\mathbf{1}$ & $\mathbf{1 6 3}$ & 2 \\
\hline 5 & 1 & 1 & $(31 u 311331311003 u 0 u 1 u 0 u 1 u 331 u 1 u u 1)$ & $\mathbf{1}$ & $\mathbf{1 6 5}$ & 2 \\
\hline 6 & 1 & $u+1$ & $(u 333010 u 1 u 33 u 011 u 33 u 03 u 100101130)$ & $\mathbf{1}$ & $\mathbf{1 6 7}$ & 2 \\
\hline 7 & 1 & $u+1$ & $(333010130 u u 00 u 10130101 u 30 u 1 u 1133)$ & $\mathbf{1}$ & $\mathbf{1 6 9}$ & 2 \\
\hline 8 & 1 & 1 & $(33000303 u u 1 u 3030100 u u 101333 u 1111)$ & $\mathbf{1}$ & $\mathbf{1 7 1}$ & 2 \\
\hline 9 & 3 & $u+1$ & $(1 u 031 u 0 u 3310030 u 13 u u 3330301 u 30 u u)$ & $\mathbf{2}$ & $\mathbf{1 3 8}$ & 2 \\
\hline
\end{tabular}

\subsection{Constructions coming from groups of order 20}

Here we present the results for the above construction using $G \in\left\{C_{20}, D_{20}\right\}$. We construct self-dual codes of length 48 by considering this construction over $\mathbb{F}_{2}$.

Table 9: Binary self-dual code of length 48 from $C_{20}$.

\begin{tabular}{cccc}
\hline$\left(a_{1}, \ldots, a_{8}\right)$ & $\left(\alpha_{1}, \ldots, \alpha_{20}\right)$ & $\mid$ Aut $(C) \mid$ & Type \\
\hline$(0,0,0,0,0,1,1,1)$ & $(0,0,0,0,0,0,0,0,1,1,0,0,0,0,0,0,0,1,0,1)$ & $2^{18} \cdot 3^{2} \cdot 5^{2}$ & {$[48,24,8]$} \\
\hline$(0,0,0,0,0,1,1,1)$ & $(0,0,0,0,0,0,0,0,1,1,1,1,0,1,1,1,1,0,0,0)$ & $2^{6} \cdot 3 \cdot 5$ & {$[48,24,8]$} \\
\hline$(0,0,0,0,0,1,1,1)$ & $(0,0,0,0,0,0,1,1,1,1,1,0,1,1,1,1,1,0,1,1)$ & $2^{8} \cdot 3^{3} \cdot 5$ & {$[48,24,8]$} \\
\hline$(0,0,0,0,0,1,1,1)$ & $(0,0,0,1,1,0,0,1,0,1,1,1,1,1,0,1,1,1,0,1)$ & $2^{3} \cdot 5$ & {$[48,24,8]$} \\
\hline$(0,0,0,0,0,1,1,1)$ & $(0,0,0,1,1,0,1,0,1,0,0,0,1,0,1,1,0,0,1,0)$ & $2^{6} \cdot 3^{2} \cdot 5$ & {$[48,24,8]$} \\
\hline$(0,0,0,0,0,1,1,1)$ & $(0,0,0,1,1,1,1,0,1,1,0,0,1,0,1,1,1,1,1,0)$ & $2^{7} \cdot 3 \cdot 5$ & {$[48,24,8]$} \\
\hline
\end{tabular}

Table 10: Binary self-dual code of length 48 from $D_{20}$.

\begin{tabular}{cccc}
\hline$\left(a_{1}, \ldots, a_{8}\right)$ & $\left(\alpha_{1}, \ldots, \alpha_{20}\right)$ & $\mid$ Aut $(C) \mid$ & Type \\
\hline$(0,0,0,0,0,1,1,1)$ & $(0,0,0,0,0,0,0,0,0,0,0,0,0,1,1,0,0,1,0,1)$ & $2^{18} \cdot 3^{2} \cdot 5^{2}$ & {$[48,24,8]$} \\
\hline$(0,0,0,0,0,1,1,1)$ & $(0,0,0,0,0,0,0,0,1,1,0,0,0,1,1,1,1,1,0,1)$ & $2^{6} \cdot 3 \cdot 5$ & {$[48,24,8]$} \\
\hline$(0,0,0,0,0,1,1,1)$ & $(0,0,0,0,0,0,1,1,1,1,0,1,1,1,1,1,1,0,1,1)$ & $2^{8} \cdot 3^{3} \cdot 5$ & {$[48,24,8]$} \\
\hline$(0,0,0,0,0,1,1,1)$ & $(0,0,0,1,1,0,0,1,0,1,0,0,0,1,1,1,1,0,0,0)$ & $2^{6} \cdot 3^{2} \cdot 5$ & {$[48,24,8]$} \\
\hline$(0,0,0,0,0,1,1,1)$ & $(0,0,0,1,1,0,0,1,0,1,0,1,1,1,1,1,1,0,1,1)$ & $2^{7} \cdot 3 \cdot 5$ & {$[48,24,8]$} \\
\hline$(0,0,0,0,0,1,1,1)$ & $(0,0,0,1,1,0,1,0,0,1,0,0,0,1,1,0,1,1,0,0)$ & $2^{3} \cdot 5$ & {$[48,24,8]$} \\
\hline
\end{tabular}




\section{Conclusion}

In this work, we introduce a new construction (quadruple border) together with certain group rings for constructing self-dual codes. Additionally, we established a link between certain elements of the group ring (units and non-units) and self-dual codes under this construction. We establish the relevance of this new construction by constructing new extremal self-dual codes of length 68 . In particular, we construct the following unknown $W_{68,2}$ codes:

$$
\begin{aligned}
& (\gamma=0, \quad \beta=\{159,163\}) \\
& (\gamma=1, \quad \beta=\{44,48,161,163,165,167,169,171\}) \\
& (\gamma=2, \quad \beta=\{58,138,141\}) \\
& (\gamma=3, \quad \beta=\{82,86,87,89,92,99,111\}) \text { and } \\
& (\gamma=4, \quad \beta=\{100,114,139,143,149,158,162,166,167,168,169,170,171\}) .
\end{aligned}
$$

We consider certain groups of order $4 p$ where $p$ is odd. A suggestion for further work would be to consider other classes of groups of order $4 p$ and possibly groups of order $4 p$ when $p$ is even. Finally, one could consider groups of order larger than 20.

\section{References}

[1] F. Bernhardt, P. Landrock, and O. Manz, The extended Golay codes considered as ideals, J. Combin. Theory Ser. A, 55, no. 2, 1990, 235 - 246.

[2] S. Buyuklieva, I. Boukliev, Extremal self-dual codes with an automorphism of order 2, IEEE Trans. Inform. Theory, 44, 1998, 323 - 328.

[3] C.L. Chen, W.W. Peterson, E.J. Weldon, Some results on quasi-cyclic codes, Information and Control, 15, 1969, 407-423.

[4] J.H. Conway and N.J.A. Sloane, A new upper bound on the minimal distance of selfdual codes, IEEE Trans. Inform. Theory, 36, no. 6, 1990, 1319 - 1333.

[5] P. J. Davis, Circulant Matrices, Chelsea Publishing New York, 1979.

[6] S.T. Dougherty, Algebraic Coding Theory Over Finite Commutative Rings, SpringerBriefs in Mathematics. Springer, Cham, 2017, ISBN: 978-3-319-59805-5; 978-3-31959806-2.

[7] S.T. Dougherty, P. Gaborit, M. Harada and P. Sole, Type II codes over $\mathbb{F}_{2}+u \mathbb{F}_{2}$, IEEE Trans. Inform. Theory, 45, 1999, 32 - 45. 
[8] S.T. Dougherty, J. Gildea, R. Taylor, A. Tylshchak, Group rings, G-codes and constructions of self-dual and formally self-dual codes, Des. Codes Cryptogr., 86, no 9, $2018,2115-2138$.

[9] S.T. Dougherty, J. Gildea, A. Korban, A. Kaya, A. Tylshchak, B. Yildiz, Bordered constructions of self-dual codes from group rings, submitted.

[10] S.T. Dougherty, M. Harada, T.A. Gulliver, Extremal binary self-dual codes, IEEE Trans. Information Theory, 43, no. 6, 1997, 2036 - 2047.

[11] S.T.Dougherty, J.-L. Kim, H. Kulosman, H. Liu, Self-dual codes over commutative Frobenius rings, Finite Fields Appl., 16, 2010, 14 - 26.

[12] S.T. Dougherty, B. Yildiz, S. Karadeniz, Codes over $R_{k}$, Gray maps and their binary images, Finite Fields Appl., 17, no. 3., 2011, 205 - 219.

[13] S.T. Dougherty, B. Yildiz, S. Karadeniz, Self-dual codes over $R_{k}$ and binary self-dual codes, European Journal of Pure and Applied Mathematics, 6, no. 1, 2013, 89 - 106.

[14] P. Gaborit, V. Pless, P. Sole and O. Atkin, Type II codes over $\mathbb{F}_{4}$, Finite Fields Appl., 8, no. 2, 2002, 171 - 183.

[15] J. Gildea, A. Kaya, R. Taylor, B. Yildiz, Constructions for self-dual codes induced from group rings, Finite Fields Appl., 51, 2018, 71 - 92.

[16] T.A. Gulliver, M. Harada, Weight enumerators of double circulant codes and new extremal self-dual codes, Des. Codes Cryptogr., 11, no. 2, 2997, 141 - 150.

[17] T.A. Gulliver, M. Harada, Classification of extremal double circulant formally self-dual even codes, Des. Codes Cryptogr., 11, no. 1, 1997, 25 - 35.

[18] T.A. Gulliver, M. Harada, H. Miyabayashi, Double circulant and quasi-twisted self-dual codes over $\mathbb{F}_{5}$ and $\mathbb{F}_{7}$, Adv. Math. Commun., 1, no. 2, 2007, 223 - 238.

[19] T. A. Gulliver, M. Harada, On double circulant doubly even self-dual $[72,36,12]$ codes and their neighbors, Australas. J. Combin., 40, 2008, 137 - 144.

[20] T.A. Gulliver, M. Harada, On the performance of optimal double circulant even codes, Adv. Math. Commun., 11, no. 4, 2017, 767 - 775.

[21] M. Harada, A. Munemasa, Some restrictions on weight enumerators of singly even self-dual codes, IEEE Trans. Inform. Theory 52, 2006, 1266 - 1269.

[22] T. Hurley, Group Rings and Rings of Matrices, Int. Jour. Pure and Appl. Math, 31, no. 3, 2006, $319-335$. 
[23] T. Hurley, Self-dual, dual-containing and related quantum codes from group rings, arXiv:0711.3983, 2007.

[24] A. Kaya, B. Yildiz, A. Pasa, New extremal binary self-dual codes from a modified four circulant construction, Discrete Math., 339, no. 3, 2016, 1086 - 1094.

[25] M. Karlin, New binary coding results by circulants, IEEE Trans. Information Theory 15, 1969, $81-92$.

[26] F.J. MacWilliams, N.J.A. Sloane, The theory of error-correcting codes. I. NorthHolland Mathematical Library, 16, North-Holland Publishing Co., Amsterdam-New York-Oxford, 1977. pp. i-xv and 1 - 369. ISBN: 0-444-85009-0.

[27] W. Bosma, J. J. Cannon, C. Fieker, A. Steel (eds.), Handbook of Magma functions, Edition 2.16 (2010), 5017 pages.

[28] S. Ling and P. Sole, Type II codes over $\mathbb{F}_{4}+u \mathbb{F}_{4}$, Europ. J. Combinatorics, 22, 2001, $983-997$.

[29] I. McLoughlin, A group ring construction of the [48, 24, 12] Type II linear block code, Des. Codes Cryptogr., 63, no. 1, 2012, 29 - 41.

[30] McLoughlin, I., Hurley, T., A group ring construction of the extended binary Golay code, IEEE Trans. Inform. Theory, 54, no. 9, 2008, 4381 - 4383.

[31] E.M. Rains, Shadow bounds for self-dual codes, IEEE Trans. Inform. Theory, 44, 1998, $134-139$. 